\title{
Session VII: Head and neck
}

\section{Oncology keynote: Overview of chemoprevention in head and neck squamous cell cancer: Molecular screening and risk prediction}

Vali Papadimitrakopoulou

University of Texas MD Cancer Center, Thoracicl Health \& Neck Medical Oncology, Houston, TX 77030, USA

Although the role of tobacco and alcohol consumption in the etiology of head and neck squamous cell cancer (HNSCC) is well accepted, the genetic alterations that lead to invasive cancer formation are incompletely understood. The field of chemoprevention emerged from the preclinically and epidemiologically based premise that carcinogenesis, in early, premalignant stages, is reversible. Increasing interest in identifying candidate biomarkers that could serve as risk predictors has produced several candidates.

Among the most significant are genetic instability in the form of chromosomal polysomy [1-3] and aneuploidy, cyclin D1 [4] and epidermal growth factor receptor (EGFR) [5-7], allelic losses at $3 p$ and $9 p$ [812] and p53 alterations [13]. Current chemoprevention studies involve interventions targeted toward p53 abnormalities, as well as selective cyclooxygenase- 2 inhibitors, natural products such as green tea extract, and EGFR kinase inhibitors. Despite progress in chemoprevention [14], the approval of agents in this setting has been slow, emphasizing the need to focus on molecular characterization of the epithelial field and develop a clear risk model to be used as the basis for new selectively targeted intervention.

\section{References}

[1] J.S. Lee, S.Y. Kim, W.K. Hong et al., Detection of chromosomal polysomy in oral leukoplakia, a premalignant lesion, $J$ Nat Cancer Inst 85 (1993), 1951-1954.

[2] N. Voravud, D.M. Shin, J.Y. Ro et al., Increased polysomies of chromosomes 7 and 17 during head and neck multistage tumorigenesis, Cancer Res 53 (1993), 2874-2883.
[3] J.A. Veltman, F.J. Bot, F.C. Huynen et al., Chromosome instability as an indicator of malignant progression in laryngeal mucosa, J Clin Oncol 18 (2000), 1644-1651.

[4] J.G. Izzo, V. Papadimitrakopoulou, D.D. Liu et al., Cyclin D1 genotype, response to biochemoprevention, and progression rate to upper aerodigestive tract cancer, J Natl Cancer Inst 95(3) (2003), 198-205.

[5] J.R. Grandis and D.J. Tweardy, Elevated levels of transforming growth factor alpha and epidermal growth factor receptor messenger RNA are early markers of carcinogenesis in head and neck cancer, Cancer Res 53 (1993), 3579-3584.

[6] J.R. Grandis, D.J. Tweardy and M.F. Melhem, Asynchronous modulation of transforming growth factor alpha and epidermal growth factor receptor protein expression in progression of premalignant lesions to head and neck squamous cell carcinoma, Clin Cancer Res 4 (1998), 13-20.

[7] D.M. Shin, J.Y. Ro, W.K. Hong et al., Dysregulation of epidermal growth factor receptor expression in premalignant lesions during head and neck tumorigenesis, Cancer Res 54 (1994), 3153-3159.

[8] P. van der Riet, H. Nawroz, R.H. Hruban et al., Frequent loss of chromosome 9p21-22 early in head and neck cancer progression, Cancer Res 54 (1994), 1156-1158.

[9] M. Partridge, G. Emilion, S. Pateromichelakis et al., Allelic imbalance at chromosomal loci implicated in the pathogenesis of oral precancer, cumulative loss and its relationship with progression to cancer, Oral Oncol 34 (1998), 77-83.

[10] L. Mao, J.S. Lee, Y.H. Fan et al., Frequent microsatellite alterations at chromosomes 9p21 and 3p14 in oral premalignant lesions and their value in cancer risk assessment, Nat Med $\mathbf{2}$ (1996), 682-685.

[11] M. Partridge, S. Pateromichelakis, E. Phillips et al., A casecontrol study confirms that microsatellite assay can identify patients at risk of developing oral squamous cell carcinoma within a field of cancerization, Cancer Res 60 (2000), 3893 3898.

[12] M.P. Rosin, X. Cheng, C. Poh et al., Use of allelic loss to predict malignant risk for low-grade oral epithelial dysplasia, Clin Cancer Res 6 (2000), 357-62.

[13] J.A. Brennan, L. Mao, R.H. Hruban et al., Molecular assessment of histopathological staging in squamous-cell carcinoma of the head and neck, N Engl J Med 332 (1995), 429-435.

[14] W.K. Hong and M.B. Sporn, Recent advances in chemoprevention of cancer, Science 278 (1997), 1073-1077. 


\section{Genetic instability: A driving force and risk biomark- er for head and neck cancer}

\author{
Walter N. Hittelman \\ University of Texas MD Anderson Cancer Center, \\ Houston, TX 77030
}

Tumorigenesis in the upper aerodigestive tract represents a field cancerization process where epithelium is exposed to carcinogenic insult (e.g., tobacco) and cofactors (e.g., alcohol), resulting in chronic tissue damage and wound repair. Using chromosome in situ hybridization techniques to quantify the levels of genetic changes in head and neck tumors and their adjacent epithelium, cells with abnormal chromosome copy numbers are detected in normal-appearing epithelium within the head and neck cancer field; the level of chromosome polysomy increases with histological progression to cancer. Spatial genetic mapping studies indicate a process of random chromosome instability and multifocal clonal outgrowth throughout the epithelium at cancer risk. Chromosome instability is also detected in oral and laryngeal biopsies from leukoplakias. Individuals exhibiting high levels of chromosome instability have a high rate of subsequent cancer development. Overexpression of cyclin D1 and p53 alteration in the epithelium is associated with increased chromosome instability and increased cancer risk, while modulation of cyclin D1 levels during chemopreventive trials is associated with decreased chromosome instability and relatively delayed onset of head and neck cancer. These studies suggest that genetic instability is a driving force for head and neck tumorigenesis. Targeting the processes that promote genetic instability may be useful in slowing progression to head and neck cancer. Assessing the levels of ongoing genetic instability and clonal outgrowth may therefore be useful for identifying individuals at high cancer risk and for monitoring the impact of chemopreventive intervention.

\section{The rapid adoption of optical imaging of oral pre- malignant lesions}

Calum MacAulay ${ }^{\mathrm{a}}$, Pierre Lane ${ }^{\mathrm{a}}$, Catherine Poh $^{\mathrm{b}}$, Haishan Zeng ${ }^{\mathrm{a}}$ and Miriam Rosin ${ }^{\mathrm{a}}$

${ }^{a}$ British Columbia Cancer Research Centre, Research Arm of the BC Cancer Agency, Vancouver, BC, Canada ${ }^{\mathrm{b}}$ University of British Columbia, Faculty of Dentistry, Vancouver, BC, Canada

Optical interrogation of tissue in vivo is one of the earliest of diagnostic techniques. As we improve our understanding of the ways in which light interacts with tissue, and in particular with the structural and molecular changes of neoplasia in the oral cavity, simple but effective optical diagnostic tools or biopsy guidance devices can be designed. Also, as our knowledge has improved of how light interacts with interrogated tissue structural and molecular properties, the pace of clinical development and adoption has increased remarkably. Correlating measured optical properties with not only tissue histopathological interpretation but with molecular changes and genetic damage has increased the acceptance of these photonic tools. Conventional histopathological tissue interpretation results are compared with molecular-based risk assessment, and observable optical changes contrasted with measured molecular and structural tissue changes. 